\title{
In Vivo Functional and Myeloarchitectonic Mapping of Human Primary Auditory Areas
}

\author{
Frederic Dick, ${ }^{1,2}$ Adam Taylor Tierney, ${ }^{4}$ Antoine Lutti, ${ }^{5}$ Oliver Josephs, ${ }^{1,5}$ Martin I. Sereno, ${ }^{1,2,3 \star}$ \\ and Nikolaus Weiskopf ${ }^{5 *}$ \\ ${ }^{1}$ Birkbeck/UCL Centre for NeuroImaging, London WC1E 7HX, United Kingdom; ${ }^{2}$ Department of Psychological Sciences, Birkbeck College, University of \\ London, London WC1E 7HX, United Kingdom; ${ }^{3}$ Perceptual and Language Sciences Division, University College London, London WC1E 6BT, United \\ Kingdom; ${ }^{4}$ Northwestern University, Evanston, Illinois 60208; and ${ }^{5}$ Wellcome Trust Centre for Neuroimaging, Institute of Neurology, University College \\ London, London WC1N 3BG, United Kingdom
}

In contrast to vision, where retinotopic mapping alone can define areal borders, primary auditory areas such as A1 are best delineated by combining in vivo tonotopic mapping with postmortem cyto- or myeloarchitectonics from the same individual. We combined highresolution $(800 \mu \mathrm{m})$ quantitative $\mathrm{T}_{1}$ mapping with phase-encoded tonotopic methods to map primary auditory areas (A1 and $\left.\mathrm{R}\right)$ within the "auditory core" of human volunteers. We first quantitatively characterize the highly myelinated auditory core in terms of shape, area, cortical depth profile, and position, with our data showing considerable correspondence to postmortem myeloarchitectonic studies, both in cross-participant averages and in individuals. The core region contains two "mirror-image" tonotopic maps oriented along the same axis as observed in macaque and owl monkey. We suggest that these two maps within the core are the human analogs of primate auditory areas A1 and R. The core occupies a much smaller portion of tonotopically organized cortex on the superior temporal plane and gyrus than is generally supposed. The multimodal approach to defining the auditory core will facilitate investigations of structure-function relationships, comparative neuroanatomical studies, and promises new biomarkers for diagnosis and clinical studies.

\section{Introduction}

The parcellation of cortex into distinct areas is a long-standing program in neuroscience (Zilles and Amunts, 2010), but one more advanced in vision than in audition. While some early visual areas can be defined solely on the basis of architectonics (e.g., stria in V1) or noninvasive retinotopy (Engel et al., 1994), the borders of primary auditory cortex (A1) cannot easily be defined by anatomy or function alone. Areas $\mathrm{A} 1$ and $\mathrm{R}$ are two major fields of the "auditory core" (Hackett, 2007), a narrow, keyholeshaped region on the temporal plane. In ex vivo preparations, auditory core shows heavy, layer IIIb/IV-specific staining for cell bodies, myelin, acetylcholinesterase, cytochrome oxidase, and parvalbumin (Hackett, 2011). Although greater myelination in caudal core may correspond to A1 (Hackett et al., 2001), the boundary with $\mathrm{R}$ is difficult to define using myeloarchitectonic criteria (Morel et al., 1993).

\footnotetext{
Received April 6, 2012; revised Aug. 21, 2012; accepted Sept. 14, 2012.

Author contributions: F.D., A.T.T., A.L., M.I.S., and N.W. designed research; F.D., A.T.T., and A.L. performed research; 0.J. contributed unpublished reagents/analytic tools; F.D., A.L., and M.I.S. analyzed data; F.D., M.I.S., and N.W. wrote the paper.

This research was funded by the Medical Research Council G0400341 and G0700399, The Royal Society RG081218, Royal Society Wolfson Research Merit Award, and National Institutes of Health R01 MH 081990. The Wellcome Trust Centre for Neuroimaging is supported by core funding from the Wellcome Trust 091593/Z/10/Z. We thank Troy Hackett, Lori Holt, Jen Linden, and Sam Schwarzkopf for very useful comments and suggestions, and Holly Bridge and Stuart Clare for generous support.

${ }^{*}$ M.I.S. and N.W. contributed equally to this work.

Correspondence should be addressed to Frederic Dick, Birkbeck/UCL Centre for Neurolmaging, Birkbeck College, Malet Street, London, WC1E 7HX. E-mail: f.dick@bbk.ac.uk.

DOI:10.1523/JNEUROSCI.1712-12.2012

Copyright $\odot 2012$ the authors $\quad 0270-6474 / 12 / 3216095-11 \$ 15.00 / 0$
}

A1 and R are challenging to delineate using tonotopic mapping since tonotopy provides only one spatial axis, with no agreed upon means to define borders perpendicular to isofrequency bands, which span multiple auditory areas (Hackett, 2011). In a small number of invasive experiments in animals, physiological measures (tonotopy, response properties) have been combined with ex vivo histological mapping to define borders (Merzenich and Brugge, 1973; Imig et al., 1977; Pfingst and O'Connor, 1981; Morel and Kaas, 1992; Morel et al., 1993).

Studies of anesthetized macaques show best frequency (BF) progressions across and around auditory core. As seen in Figure 1 (most complete map from Morel et al., 1993, redrawn and contoured), A1-in the highly myelinated caudomedial (CM) core-is characterized by a high-to-low BF progression, from a medial high BF corona descending to a rostrolateral low BF trough, which marks the A1/R border. In the narrower and more lightly myelinated area $\mathrm{R}$ in the lateral aspect of the core, there is a gentler low-to-mid BF progression moving rostrolaterally.

There are multiple tonotopic maps across the temporal plane, as shown with functional magnetic resonance imaging (fMRI) in humans (Formisano et al., 2003; Talavage et al., 2004; Da Costa et al., 2011) and macaques (Petkov et al., 2006; Baumann et al., 2010). Whereas the A1/R boundary can be localized using tonotopic gradients (Formisano et al., 2003), the relation between frequency progressions and the position of the core is disputed (Humphries et al., 2010). Current probabilistic postmortem cytoarchitectonic maps of primary auditory areas (Morosan et al., 2001) are too blurry to localize core boundaries. 
Anatomical MRI results (Walters et al., 2003; Bridge and Clare, 2006; Sigalovsky et al., 2006; Trampel et al., 2011; Glasser and Van Essen, 2011; Sereno et al., 2012) suggest that areal boundaries can be defined by measuring proxies for myelination. Here, we characterize the strongly myelinated auditory core using a highresolution bias-free quantitative $\mathrm{T}_{1}$ mapping protocol that provides estimates of myelination directly comparable across cortical regions or subjects. We combine these myelin maps with detailed tonotopic mapping in the same participants to characterize frequency progressions within and around auditory core.

\section{Materials and Methods}

Participants. Six adults (ages 22-55, three female) with normal hearing and vision participated in all parts of the study. Three additional adults (ages 22-30, all male) participated only in the functional imaging part of the study. Experimental protocols were approved by local ethics committees, and all participants gave informed and signed written consent.

Structural imaging. Structural images were acquired on a whole-body Tim Trio system (2.89 T; Siemens Healthcare), with radiofrequency (RF) body transmit and 32-channel receive head coil at the Wellcome Trust Centre for Neuroimaging. $3 \mathrm{~T}$ scanners such as the Trio will not have as high signal-to-noise ratio (SNR) as higher field strength (e.g., $7 \mathrm{~T}$ ), but do benefit from greater uniformity of signal (less $B_{1}$ field inhomogeneities), smaller susceptibility artifacts ( $B_{0}$ field inhomogeneities), lower acoustic noise, and less dropout in parts of the temporal lobe due primarily to $B_{1}$ field inhomogeneities. As part of the quantitative $\mathrm{R}_{1}$ mapping protocol, proton density-weighted (PDw) and $\mathrm{T}_{1}$-weighted $\left(\mathrm{T}_{1} \mathrm{w}\right)$ images were acquired using an in-house multi-echo 3D FLASH pulse sequence (Weiskopf et al., 2011): voxel size: $0.8 \times 0.8 \times$ $0.81 \mathrm{~mm}^{3}, \mathrm{FOV}=256 \times 216 \times 194 \mathrm{~mm}$, matrix $=320 \times 270 \times 240$, $\mathrm{TR}=23.7 \mathrm{~ms}$, excitation flip angle: $6^{\circ}(\mathrm{PDw})$ or $28^{\circ}\left(\mathrm{T}_{1} \mathrm{w}\right)$. Acquisition was sped up by $2 \times$ GRAPPA parallel imaging in the phase encoding and 6/8 Partial Fourier in the partition direction. To improve image quality (maximize SNR and minimize geometric distortion at the same time), four gradient echoes were acquired $(\mathrm{TE}=2.20,4.75,7.30,9.85 \mathrm{~ms}$ ) with high readout bandwidth after each excitation pulse. Each session consisted of four $10 \mathrm{~min} 31 \mathrm{~s}$ acquisitions (two PDw and two $\mathrm{T}_{1} \mathrm{w}$ ) and two shorter scans to estimate B0 and B1 inhomogeneities (see below). Quantitative $\mathrm{R}_{1}\left(=1 / \mathrm{T}_{1}\right)$ maps were estimated from the PDw and $\mathrm{T}_{1} \mathrm{w}$ images according to the model developed by Helms et al. (2008a) including a correction for RF transmit field inhomogeneities (Lutti et al., 2010) and imperfect spoiling (Preibisch and Deichmann, 2009). Recent applications of this method have demonstrated its robustness and accuracy (Helms et al., 2008b, 2009; Draganski et al., 2011; Weiskopf et al., 2011). To correct for the effect of RF transmit inhomogeneities on $\mathrm{R}_{1}$ maps, maps of the transmit field $\mathrm{B}^{+}{ }^{+}$were acquired using a 3D echoplanar imaging (EPI) spin-echo (SE)/stimulated echo (STE) method (FOV = $256 \times 192 \times 192 \mathrm{~mm}^{3}$, matrix $=64 \times 48 \times 48, \mathrm{TE}_{\mathrm{SE}} / \mathrm{TE}_{\mathrm{STE}}=39.38 /$ $72.62 \mathrm{~ms}, \mathrm{TR}=500 \mathrm{~ms}, \alpha$ varying from 270 to $130^{\circ}$ by steps of $10^{\circ}$, acquisition time $3 \mathrm{~min} 48 \mathrm{~s}$ ) (Lutti et al., 2012), which was corrected for off-resonance effects using a standard B0 field map (double gradient echo FLASH, $3 \mathrm{~mm}$ isotropic resolution, whole-brain coverage (Lutti et al., 2010).

Functional imaging. Functional images were acquired on a $1.5 \mathrm{~T}$ whole-body Tim Avanto System (Siemens Healthcare), at the Birkbeck/
University College London Centre for NeuroImaging, with RF body transmit and a 32-channel receive head coil. (Supplemental data for three participants was also acquired using a specialized single-loop coil positioned over the superior temporal gyrus). 1.5 T has evolved dramatically over the last years with much more stable hardware and 32-channel head coils leading to $200-300 \%$ improvement in blood oxygenation leveldependent (BOLD) sensitivity in auditory regions (Wiggins et al., 2006); EPI at this field strength also has less warping and distortion in auditory regions than higher field magnets. EPIs were acquired with the following parameters: 24 slices, voxel resolution $3.2 \times 3.2 \times 3.2 \mathrm{~mm}$ (matrix size: $64 \times 64$ ), flip angle $=90^{\circ}$, bandwidth $=1474 \mathrm{~Hz} /$ pixel, $\mathrm{TR}=2000 \mathrm{~ms}$, $\mathrm{TE}=39 \mathrm{~ms}$, data acquired with prospective motion correction (Thesen et al., 2000). The voxel resolution is similar to that $(\sim 3 \times 3 \times 3 \mathrm{~mm})$ used in many recent retinotopy studies of small areas, such as V6, VIP, FEF, IPS1\&2, V8, and LOC, e.g., Larsson and Heeger (2006), Sereno and Huang (2006), Hagler et al. (2007), Rajimehr and Tootell (2009), and Konen et al. (2011). Individual scans had 262 volumes; to allow longitudinal relaxation to reach equilibrium, six initial volumes were discarded from each run (initial 2 not saved by scanner). For each imaging session, a short (3 min) $\mathrm{T}_{1}$-weighted 3D MPRAGE (88 partitions, voxel resolution $1 \times 1 \times 2 \mathrm{~mm}^{3}$, flip angle $=7^{\circ}, \mathrm{TE}=4 \mathrm{~ms}, \mathrm{TI}=1000 \mathrm{~ms}, \mathrm{TR}=8.2$ $\mathrm{ms}$, mSENSE acceleration $=2 \times$, slab-selective excitation) was acquired with the same orientation and slice block center as the functional data for initial alignment with the high-resolution scans used to reconstruct the subject's cortical surface. For the three additional participants who did not take part in the quantitative imaging, we acquired a single highresolution $\mathrm{T}_{1}$-weighted MPRAGE for cortical surface reconstruction $\left(1 \times 1 \times 1 \mathrm{~mm}, 176\right.$ slices, $\mathrm{TR}=2730 \mathrm{~ms}, \mathrm{TE}=3.57 \mathrm{~ms}$, flip angle $\left.=7^{\circ}\right)$.

Auditory stimuli. Both retinotopic (Saygin and Sereno, 2008) and tonotopic (Woods et al., 2009) mapping studies have suggested that targeted attentional demands and increased stimulus complexity can significantly modulate fMRI activation in cortical maps. Thus, we used 
bandpass-swept complex and engaging nonlinguistic vocalizations to map tonotopic regions. Base stimuli were adapted from the Montreal Affective Voices (Belin et al., 2008), a series of recordings of emotional nonverbal vocalizations elicited by actors producing sounds corresponding to a set of eight emotions (a ninth "neutral" emotion was not used in the current study, and only male voices were included). Tokens from this set were randomly selected such that no two recordings were repeated until the entire set had been presented. These tokens were then spliced together with no intervening pause to form $8 \mathrm{~min}$, $32 \mathrm{~s}$ long passages. Next, these stimuli were amplitude compressed in Adobe Audition 1.5 with additional manual editing; a bandpass filter was then cycled with a period of $64 \mathrm{~s}$ over the entire passage with center frequency logarithmically ascending from 150 to $9600 \mathrm{~Hz}(Q=2$, expanding to $Q=3$ at tails, where $Q$ is the ratio of center frequency over bandwidth). Descending stimuli were created by reversing the entire waveform before filtering, then re-reversing at the final step. This initial filtering was cleaned using similarly ascending low- and high-pass filters positioned an octave above and below the center frequency. Finally, a dynamic amplitude envelope was imposed over the frequency sweeps to equilibrate perceived loudness in the scanner environment. For presentation with the Sensimetrics headphones, stimuli were passed through a final earbud-specific filter that compensated for the slight frequency peaks and phase offsets induced by the acoustic transfer functions of the earbuds. During scanning, subjects were asked to monitor the stimuli and press a button whenever they heard laughter (laughter stimuli were sparsely and nonperiodically distributed through the stimulus train). For an example of a single stimulus sweep, see Notes.

Additional control stimuli were created to test the generality of the tonotopic maps across different types of sound. Similar bandpass-sweep filtering procedures were used on carefully edited musical excerpts (ABBA or Beatles songs), repetitive male speech samples, or amplitude modulated $(16 \mathrm{~Hz})$ white noise (noise stimuli similar to Talavage et al., 2004).

Stimulus setups. Each subject was scanned using two different stimulus delivery setups in two to five different sessions. In the first setup, stimuli were delivered by an in-house manufactured electrodynamic, magnetless headphone (Josephs et al., 2009) using an isodynamically driven lightweight membrane either coupled to an ear insert that also acted as a passive attenuator of acoustical scanner noise, or presented directly at the ear. The headphone gives a high amplitude, smooth, and minimally distorted response over a wide bandwidth $(\sim 14 \mathrm{kHz})$. Stimuli were delivered to only the left ear, as the size of the ear defender did not allow for subjects' heads to fit into the compact 32-channel head coil with two headphones. The numbers of $8 \mathrm{~min} 32 \mathrm{~s}$ long runs collected per participant using this setup were $4,8,8,8,8,8,12,12$, and 16 . Three participants also took part in additional control stimulus conditions (see below), each with four 8 min 32 s runs.

In the second setup, stimuli were delivered binaurally using in-house safety-enhanced Sensimetrics (Malden) S14 earbuds, with the head centered within the 32-channel coil. NoMoCo (NoMoCo Inc.) cushions were placed around the head to provide additional passive scanner acoustical noise attenuation and to stabilize head position. All volunteers participated in four $8 \mathrm{~min} 32 \mathrm{~s}$ long runs in this setup. Finally, a subset of seven participants was scanned on an additional single run of an $8 \mathrm{~min}$ 32 s block design "auditory localizer" experiment where 16 s blocks of the tonotopy stimulus (with stimuli across blocks balanced for frequency range) were alternated with $16 \mathrm{~s}$ blocks of no stimulation.

Cortical surface reconstruction and sampling of $R_{1}$ values within cortical ribbon. Cortical surfaces were reconstructed with FreeSurfer (v5.0.0; Dale et al., 1999) from the aligned (AFNI 3dAllineate; Cox, 2012), handinspected average of the two high-resolution $\mathrm{T}_{1}$-weighted scans acquired for $R_{1}$ mapping. We initially attempted using quantitative $R_{1}$ scans for surface reconstruction but experienced localized segmentation failures because some boundaries between the pial surface, the CSF, and the skull have different contrast from FreeSurfer's priors in the segmentation algorithms. (Cortical surfaces for the three participants who were not scanned using quantitative protocols were reconstructed using the single $\mathrm{T}_{1}$-weighted MPRAGE acquired at $1.5 \mathrm{~T}$ ). Each subject's reconstructed cortical surface was inflated to a sphere and registered to an average spherical surface atlas in FreeSurfer using a best-fit sulcal alignment (Fischl et al., 1999).

$\mathrm{R}_{1}$ datasets were sampled along the normal to each gray/white matter surface vertex in steps of $10 \%$ of cortical thickness (thickness estimated in FreeSurfer; Fischl and Dale, 2000) and then smoothed tangentially at each depth with a $4 \mathrm{~mm}$ full-width at half-maximum (FWHM) 2D kernel. The human cortex has deep sulci, but also a complex pattern of concavity and convexity, including many convex regions buried within major sulci. Postmortem studies in humans show that myeloarchitecture varies significantly with local cortical convexity (Annese et al., 2004), where more convex regions are thicker and more myelinated, especially in middle and upper layers. Recently we found that there is a moderately strong correlation between local curvature and $\mathrm{R}_{1}$ at middle cortical depth fractions as well as a lesser but still significant correlation between cortical thickness and $\mathrm{R}_{1}$ (Sereno et al., 2012). Thus, for each participant, we used the FreeSurfer estimate of local curvature (smoothed with a $<1$ mm FWHM 2D kernel) and cortical thickness as hemisphere-wise linear predictors of $R_{1}$ values at each cortical depth to adjust for curvaturedependent changes in myelination as well as potential sampling artifacts due to local imperfections in reconstructed white or pial surfaces. Vertexwise residuals from this regression were used as "de-curved" and "dethickened" estimates of $\mathrm{R}_{1}$ values whose units are directly comparable to raw demeaned $\mathrm{R}_{1}$ values. Cross-subject cortical surface-based averages of $\mathrm{R}_{1}$-derived values were calculated by projecting the values from each subject onto the unit sphere after 1 step $(<1 \mathrm{~mm}$ FWHM) of surfacebased smoothing (Hagler et al., 2006), and averaging the values at each vertex. For visualization purposes, resulting average values were backprojected onto a single representative subject's entire inflated hemisphere or a resected temporal lobe showing the pial surface, with the latter graphically edited to remove the medial temporal lobe and aspects of the insula that were difficult to remove cleanly in FreeSurfer.

Creation of isofrequency contours. These data (Morel et al., 1993, their Fig. 2a) were chosen for being (to our knowledge) the most complete and representative published tonotopic physiological recordings in a single macaque where auditory core was also characterized cyto- and myeloarchitectonically. Each BF data point along the physiological tracks in Figure $2 a$ was entered into a 2D grid using the Draw Dataset plug-in in AFNI (Cox, 2012) with a high-resolution jpeg of the original figure as an underlay. The sparse data were trilinearly interpolated onto a regular high-resolution grid using the "TriScatteredInterp" function in MATLAB R2010a (MathWorks); "contourf" was used to calculate and draw logarithmically spaced isofrequency contours over the entire recording area (for a similar approach, see Recanzone et al., 1999). Coronal slice profiles and areal borders were traced directly over the jpeg in Pages '09 (Apple).

Analysis of $R_{1}$ regional differences and depth profiles using morphed probabilistic maps. To provide an initial assessment of $\mathrm{R}_{1}$-based myelination maps, and to examine $\mathrm{R}_{1}$ cortical depth profiles using an independent set of regions of interest (ROIs), we created ROIs based on the 3D cytoarchitectonic probabilistic maps of subdivisions of Brodmann's area 41 (TE1.0, TE1.1, and TE1.2) with TE1.0 corresponding to the auditory core (Hackett et al., 2001; Morosan et al., 2001, pg 695). The Morosan et al. (2001) raw probability maps provided in the current AFNI distribution (Cox, 2012) were projected to a FreeSurfer "fsaverage" brain registered to the Talairach target brain, resampled onto the cortical surface, and thresholded at $p>0.60$ to create ROI labels. The labels were $\sim 2 \mathrm{~mm}$ FWHM (five steps) surface smoothed with manual removal of isolated marked vertices (due to "spillover" from the $3 \mathrm{D}$ to $2 \mathrm{D}$ projection within the lateral fissure), then spherically morphed to each subject. The morphed (unthresholded) probability maps are illustrated in Figure $3 e$.

For each subject, average $\mathrm{R}_{1}$ values for each ROI (TE1.0, TE1.1, and TE1.2) were calculated in $10 \%$ steps of cortical thickness fraction (see above); these values were used to create $R_{1}$ depth profiles. Planned matched pairs $t$ tests comparing $\mathrm{R}_{1}$ over the three ROIs were calculated from the data sampled at $50 \%$ of cortical thickness.

Functional analyses. Functional images were motion corrected (AFNI 3 dvolreg, heptic interpolation), and hand registered $(4 \times 4$ affine) with the high-resolution average $\mathrm{T}_{1}$-weighted volume used to create the cortical surface. Initial registration was performed with the "align" $\mathrm{T}_{1}$ - 


\section{a Average Rl vs cortical depth in probabilistic ROls of auditory core}

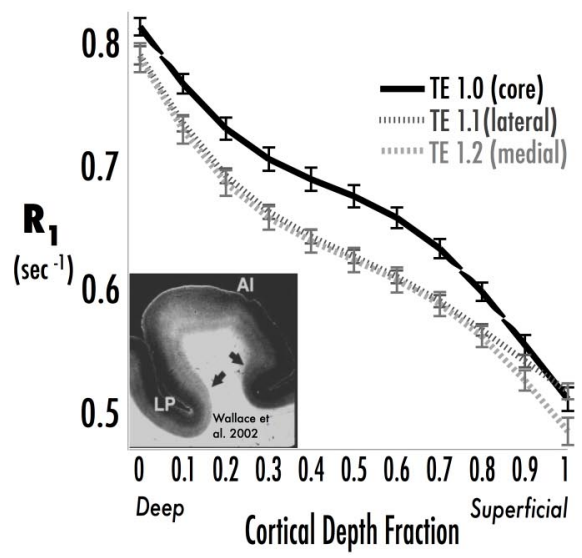

b Cross-subject-average $R_{1}$ values sampled at $50 \%$ of cortical depth onto inflated cortical surface

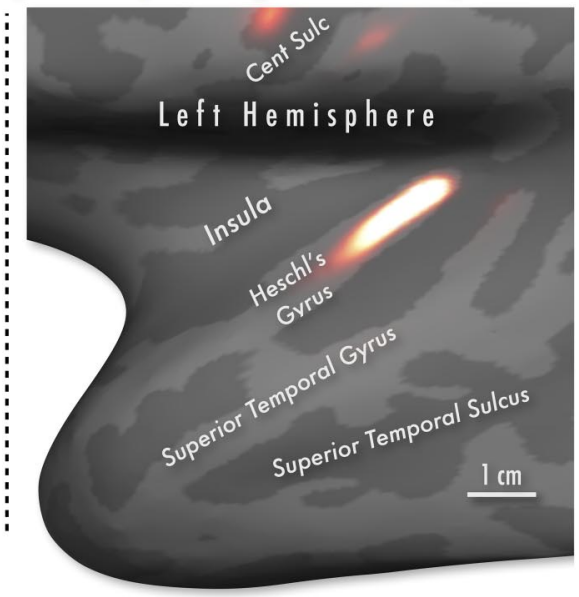

Figure 2. $\quad \boldsymbol{a}$, Left, Relaxation rate $\left(\mathrm{R}_{1} \mathrm{sec}^{-1}\right)$ as function of cortical depth, averaged within probabilistically defined subdivisions of Brodmann's area 41 (TE1.0, TE1.1, and TE1.2 according to Morosan et al., 2001). Average $R_{1}$ within TE1.0 (putative auditory core) decreases steeply from the gray/white boundary (depth fraction 0.0 ) to a tilted plateau at middle depths ( $0.3-0.6$ ), then again drops steeply at superficial depths (0.7-1.0). Error bars indicate \pm 1 SEM over subjects. $R_{1}$ within lateral (TE1.1) and medial (TE1.2) subdivisions shows a more gentle monotonic decrease from deep to superficial cortex. For comparison, the left inset is a myelin-stained section of human auditory core and belt cortex (from Wallace et al., 2002, contrast reversed) with a similar profile of myelination. $\boldsymbol{b}$, Right, Group spherical average $\mathrm{R}_{1}$ values sampled at $50 \%$ of cortical depth and projected onto a single subject's left and right inflated hemispheric surfaces. The auditory core is visible in both hemispheres as a keyhole-shaped hyperintensity maximum running posteromedially to anterolaterally over the medial half of Heschl's gyrus. Hyperintensity maxima can also be observed within the densely myelinated pre- and post-central gyri.

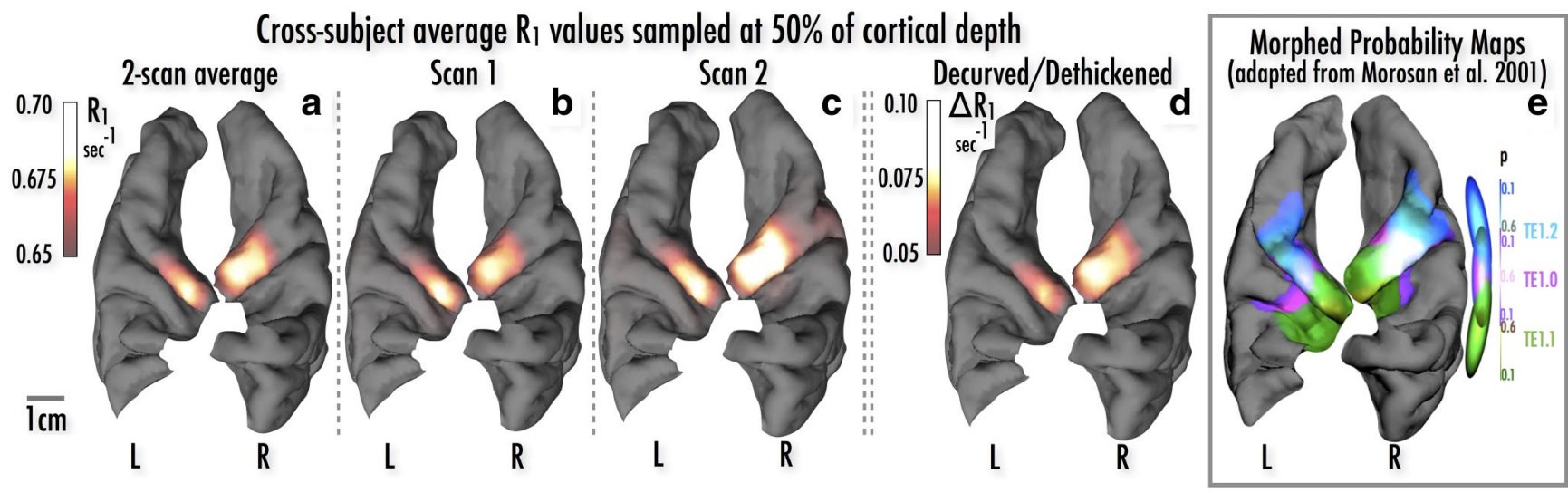

Figure 3. Group average $R_{1}$ values from $50 \%$ of cortical depth, projected onto the pial surface of the digitally resected temporal lobes of a single subject. $a$, Local increases in $R_{1}$ values along medial Heschl's gyrus, averaged across both scans, same data as Figure $2 b . \boldsymbol{b}, \boldsymbol{c}$, Single-scan $R_{1}$ averages show excellent scan-rescan reproducibility. $\boldsymbol{d}$, Maps of the change in $R_{1}$ after removing $R_{1}$ variance accounted for by local curvature and thickness show a very similar topology to raw $R_{1}$ maps. e. Probability maps of cytoarchitectonically defined TE1.0 ("core"), TE1.1, and TE1.2. derived from Morosan et al., 2001. Note that the overlap between probability distributions for TE1.0/TE1.1 and TE1.0/TE1.2 causes some probability maxima for TE1.1 (medial) and TE1.2 (lateral) to be darker colored, as shown in the overlapping probability ovoids at right.

weighted scan (same block center, slice plane direction as EPI scans); this initial registration was applied to the EPI volume, where final registration was finely adjusted by manual blink comparison (with contrast-reversed EPI images) to achieve a more exact overlay. Mapping data were analyzed using Fourier methods with individual and group analysis methods as previously described (Sereno et al., 1995; Sereno and Huang, 2006; Hagler et al., 2007), where voxels preferentially responding to a particular point in the stimulus cycle will show a higher amplitude at the frequency of stimulus cycling than at any other frequency. The phase of the signal, which corresponds to a particular point of the stimulus ramp, is then mapped to the color wheel, while the amplitude of the signal is mapped to the voxel's color saturation. Runs with downward frequency sweeps were time reversed and averaged with upward-swept scans to compensate for unspecified delays in the BOLD response.

Averaging of phase-encoded mapping data was performed using the methodology developed by Hagler et al. (2006) in which the real and imaginary components of the signal with respect to the stimulus ramp are averaged across subjects, preserving any phase information consistent between subjects. This was performed by projecting each participant's phase-encoded map to the FreeSurfer spherical atlas, performing one step of surface-based smoothing ( $<1 \mathrm{~mm}$ FWHM in 2D), averaging across subjects at each vertex, and then painting back onto a single subject's surface for viewing. Four cross-subject phase-encoded tonotopic averages were computed per hemisphere. Three of the four averages included only those subjects $(N=6)$ with both functional and $\mathrm{R}_{1}$ data, including separate averages for each auditory stimulus setup (binaural, left monaural) as well as a grand average over these two setups. The fourth average drew from independent data collected using different coil, headphone, and stimulus arrangements, and served to test map replicability. The left hemisphere average $(N=5)$ included data from three participants scanned with a $7 \mathrm{~cm}$ ring coil (Siemens Double Loop Array) over the left superior temporal gyrus, with monaural right-ear stimulation using bandpass-swept musical and vocalization stimuli, and two participants scanned with the 32-channel head coil and binaural stimulation using bandpass-swept vocalization stimuli. All data for the right hemisphere average $(N=4)$ were collected using binaural stimulation 
and the 32-channel coil and bandpass-swept musical, noise, or verbal stimuli. The three subjects without $\mathrm{R}_{1}$ data contributed to both left and right hemisphere averages; two subjects included in the first three averages contributed different data to the left (S6) and left and right hemisphere (S1) averages.

To show uninterrupted phase data in the same cortical locations in all participants (Da Costa et al., 2011), the cross-subject average block design auditory localizer data were used to create an independent functional mask for the tonotopic map data $(N=7,3.2 \mathrm{~mm}$ 2D FWHM of surface smoothing for sampling each subject's data to the icosahedral sphere, $4.5 \mathrm{~mm}$ 2D FWHM when rendering to a representative subject). A single contiguous patch of suprathreshold vertices on the superior temporal plane was extracted for each hemisphere (vertexwise $p<0.01$, with 3054 (right) and 3431 (left) connected vertices), then projected via spherical registration to each subject. Within this mask, group average and individual subject tonotopic phase-encoded data are displayed using a pseudocolor scale representing the systematic phase offsets in hemodynamic response corresponding to the phase of the auditory sweeps $(2.2$ $\mathrm{mm}$ 2D FWHM surface smoothing was applied to tonotopic phase maps to gently even out voxel edges).

Auditory frequency gradient directions were drawn by imposing isofrequency contours over the color phase map (20 steps per complete cycle), then overlaying arrows perpendicular to each contour line in and around the core, with arrow length spanning 1/40th of a complete phase cycle. The arrow direction thus represents the gradient direction while its length is proportional to the (logarithmic) tonotopic magnification factor (see below).

\section{Results}

$R_{1}$ as a function of cortical depth in probabilistically defined core and adjacent regions

As noted above, primate auditory core can be differentiated from surrounding belt regions by the presence of heavy myelination in deep and middle cortical depths with particularly heavy deep and middle layer staining within medial core (Hackett et al., 2001). An increase in overall myelination as well as expansion of layers 5 and 6 along the crown of human Heschl's gyrus has also been noted (Wallace et al., 2002). Our in vivo human MRI samples much more coarsely than histology $(800 \mu \mathrm{m}$ versus $\sim 20 \mu \mathrm{m})$, although we benefit from a relative super-resolution effect, in that at each vertex (whose position on the reconstructed cortical surface is at subvoxel resolution), multiple voxels are sampled using linear interpolation. Nevertheless, we expected $R_{1}$, which is proportional to the myelination level, to start high in deeper layers, drop to a moderate plateau at middle cortical depths, and then drop more steeply at superficial depth fractions. Given the continuously heavy myelination across middle layers of core (astriate profile; Hackett et al., 2001), the greatest $R_{1}$ difference between core and adjacent regions should be observed at middle cortical depths.

To test these predictions, we first created depth profiles of average $\mathrm{R}_{1}$ in auditory ROIs likely to correspond to auditory core proper (TE1.0) and to adjacent regions lateral and medial to core (TE1.1 and TE1.2) in the published postmortem probabilistic maps of Brodmann's area 41 (Morosan et al., 2001). The crosssubject cross-hemisphere average $\mathrm{R}_{1}$ depth profiles for TE1.0 in Figure $2 a$ show the predicted steep drop in $\mathrm{R}_{1}$ at the gray matter/ white matter border, followed by a tilted plateau in $R_{1}$ at middle cortical depths and a second steep drop approaching the gray matter/pial boundary. The $\mathrm{R}_{1}$ profiles for the medial (TE1.1) and lateral (TE1.2) regions are smoother and almost entirely overlapping, and clearly undershoot the TE1.0 "core" ROI profile at middle sampling depths as expected.

We verified these inter-ROI differences with hemisphere-wise paired $t$ tests comparing $\mathrm{R}_{1}$ values sampled halfway through cortex
(0.5). Here, $\mathrm{R}_{1}$ in TE1.0 was greater than in corresponding TE1.1 (left hemisphere (lh), $t_{(5)}=5.90, p<0.002$; right hemisphere $(\mathrm{rh})$, $\left.t_{(5)}=5.60, p<0.0025\right)$ and than in TE1.2 $\left(\mathrm{lh}, t_{(5)}=4.14, p<0.0090\right.$, rh, $\left.t_{(5)}=-12.51, p<0.0001\right)$, with no significant difference between TE1.1 and TE1.2 $(p>0.4)$.

\section{Shape, position, and size of auditory core}

Myeloarchitectonic studies (see Introduction) show that middle layer myelination of auditory core has a characteristic keyhole shape, with its long axis oriented diagonally on the superior temporal plane. In humans, it tends to lie along Heschl's gyrus (with darkest staining on the gyral crown; Wallace et al., 2002) but sometimes moves into the adjacent sulcus, with the broader rounder medial segment tapering to a thinner anterolateral finger (Hackett et al., 2001).

As an initial means of visualizing potential $\mathrm{R}_{1}$ differences expected to relate the topography of increased myelin content, we inspected the spherical surface-based cross-subject average maps of $R_{1}$ values sampled from voxels at middle cortical depths (depth fraction 0.5). When these average maps were thresholded at the mean $R_{1}$ value from the TE1.0 ROI $\left(0.66 \mathrm{~s}^{-1}\right)$, a clear hyperintensity appeared in both hemispheres, spanning a thin strip of cortex $(\sim 1.9 \mathrm{~cm} \times \sim 0.7 \mathrm{~cm})$ running approximately along the posteromedial two-thirds of Heschl's gyrus (Figs. 2b, 3a). The only other hyperintensities visible at this threshold were along the pre- and post-central gyri, corresponding to the strongly myelinated primary motor and somatosensory cortices (Glasser and Van Essen, 2011; Sereno et al., 2012). $\mathrm{R}_{1}$ values within this presumptive core region (Figs. 4, 6, $\mathrm{R}_{1}$ contours) were highest within a semicircular patch at its posteromedial aspect, with decreasing $\mathrm{R}_{1}$ moving anterolaterally. A second smaller disconnected hyperintensity appeared further laterally along the superior temporal gyrus.

As noted above, local $\mathrm{R}_{1}$ values, cortical myelin density, and laminar profile are known to vary with cortical thickness and local cortical curvature (Annese et al., 2004; Sereno et al., 2012), and thus may affect the $\mathrm{R}_{1}$-based localization of auditory core. As a validation measure, we compared the raw average $R_{1}$ maps to a cross-subject average of $R_{1}$ values after regressing out the effects of curvature and thickness (see Materials and Methods; Fig. $3 d$ ). This resulted in a picture of the keyhole-shaped maximum almost indistinguishable from that found with raw $R_{1}$ values. As an additional validation measure, comparison of cross-subject $R_{1}$ averages based on the two separate scan-rescan datasets also showed very close correspondence of auditory core localization (Fig. 3b,c). A hyperintense $\mathrm{R}_{1}$ strip over posteromedial Heschl's gyrus - the presumptive auditory core-could be easily identified in each participant, in 11 of 12 hemispheres (Figs. 4, 6, $\mathrm{R}_{1}$ contours). As seen in Figure 4, there are also additional regions of moderately high $R_{1}$ values in individual participants more laterally and posteriorly to the core; we speculate that these may correspond to area STA and potentially PA as identified in Wallace et al. (2002), but a conclusive homology will require directly comparable postmortem data.

\section{Tonotopic maps and frequency gradients within and around the core region}

Combined average human $R_{1}$ and tonotopic maps and gradients The relationship between average tonotopic maps and average $R_{1}$ maps (sampled at 0.5 cortical depth fraction) from both hemispheres was similar to that seen in individual experiments in nonhuman primates (Fig. 5, inset). In both hemispheres, the posteromedial end of presumptive core lies at the middle of an 


\section{All individual participants' $R_{1}$ values (S1-S6), sampled halfway through cortex, showing consistent myelination pattern corresponding to auditory core}

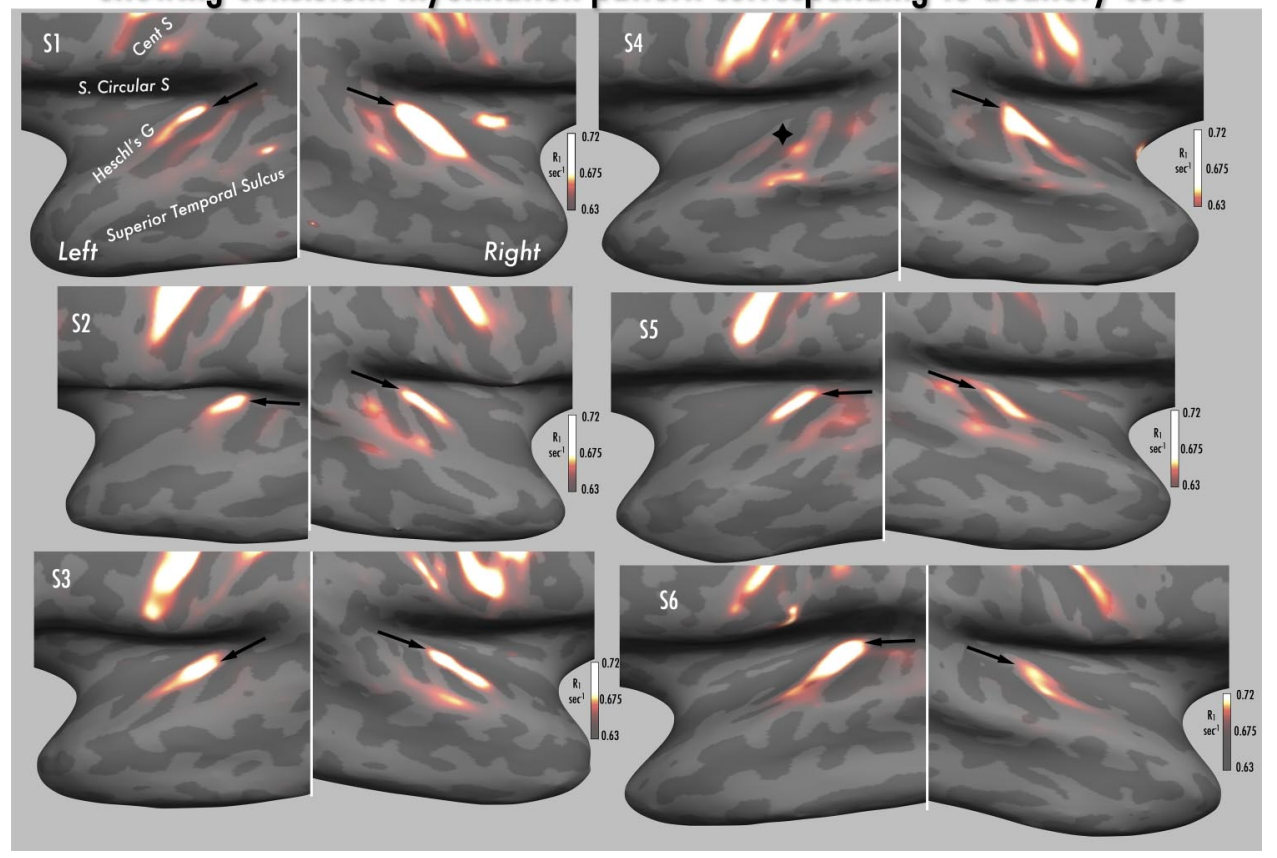

Figure 4. Montage of individual participants' $R_{1}$ maps, with values sampled halfway through cortex and projected onto the inflated surface of temporal and frontal lobes. As shown in the color bars, the range of projected $R_{1}$ values is constant over participants, with the color scale slope adjusted slightly to show individual patterns of myelination. Keyhole-shaped regional increases in $R_{1}$ oriented posteromedially to anterolaterally across Heschl's gyrus were observed in all hemispheres (medial-most aspect indicated by arrowhead) except the left hemisphere of participant $S 4$ (marked with black diamond shape). $R_{1}$ maxima are also observed along the pre- and post-central gyri within strongly myelinated presumptive primary motor and somatosensory regions. $S$. Circular $S$, superior circular sulcus of the insula; Cent S, central sulcus; Heschl's G, Heschl's gyrus.

arch-shaped high-frequency region (red). Moving anterolaterally along the long axis of the core, there is a steep high-tolow frequency progression (red $\rightarrow$ blue $\rightarrow$ green). Beyond the anterolateral end of the core, there is a large low-frequency region (green) that progresses back to high frequencies (red). On the opposite anteromedial edge of the core, selectivity progresses steeply back to high frequencies (red). Finally, at the anterior-most and posterior-most positions along the superior temporal gyrus, the characteristic frequencies descend once again (red $\rightarrow$ blue $\rightarrow$ green).

The arrangement of tonotopic maps can also be visualized by looking at the local frequency gradient direction (steepest uphill direction in frequency preference at a point; that is, $\nabla f$ with respect to cortical position). At each point, the gradient direction is perpendicular to the local isofrequency contour. The gradient direction has been drawn at a series of points along each isofrequency contour (iso-response-phase contour). The length of each vector is proportional to the scalar cortical magnification for logarithmic frequency (the reciprocal of the gradient vector amplitude). Thus, regions of slowest change in frequency sensitivity (smallest gradient amplitude) will have the longest arrows.

As noted above, the anterolateral part of the core lies within an elongated low-frequency trough in both hemispheres. Also in both hemispheres, the medial end of that low-frequency trough is located within the region with the highest myelination. From this low-frequency trough, characteristic frequencies $(\mathrm{CFs})$ rise in all directions. Along the anterolateral edge, there is a low-to-medium frequency progression, with higher CFs more lateral and anterior, as well as posterior. There is another progression from the low CF trough rising anteromedially, reaching a high CF maximum just anteromedial to the core. There is a third progression moving pos- teriorly from the middle of the core that is interrupted by a possible discontinuity, where there is an extremely rapid progression through middle frequencies that ends in a high-frequency maximum slightly posterior to the core. Finally, there is some indication of a posteromedial progression within the core in a direction parallel to the long edge that extends into higher frequency $\mathrm{CFs}$, reaching a $\mathrm{CF}$ maximum posteromedially. It is interesting that $\mathrm{CFs}$ continue to rise for a short distance beyond the posteromedial end of the core $(\sim 3 \mathrm{~mm}$; see also Individual $\mathrm{R}_{1}$ and tonotopic maps).

The pattern of tonotopic fields and progressions within and around the core was consistent over the two different experimental setups (Fig. 5, top and middle insets), although the stimulation (monaural vs binaural) and head position in the head coil (shifted to right vs centered) were different. In the right hemisphere, where auditory input was more closely matched, the average pattern of tonotopic maps over the core is highly conserved over both scan types. In the left hemisphere (ipsilateral stimulation), we also see similar tonotopic mapping, but with a shallower transition to higher characteristic frequencies in and around the posteromedial cap of the core. These tonotopic maps were also similar to cross-subject averages from data collected using different surface coil setups as well as individuals for whom $\mathrm{R}_{1}$ data were not acquired (Fig. 5, bottom insets). There was some divergence across maps (particularly medially), whereas the tonotopic progressions within and around the presumptive core region were very similar over all averages. The general layout of the tonotopic maps - where high CFs predominate along the medial superior temporal plane, with two to three high-CF "fingers" extending laterally, and interdigitate with more lateral lower CF regions extending medially — particularly paralleled the results of Formisano et al. (2003) (Fig. 5) and Humphries et al. (2010) (Figs. 5, 6). 


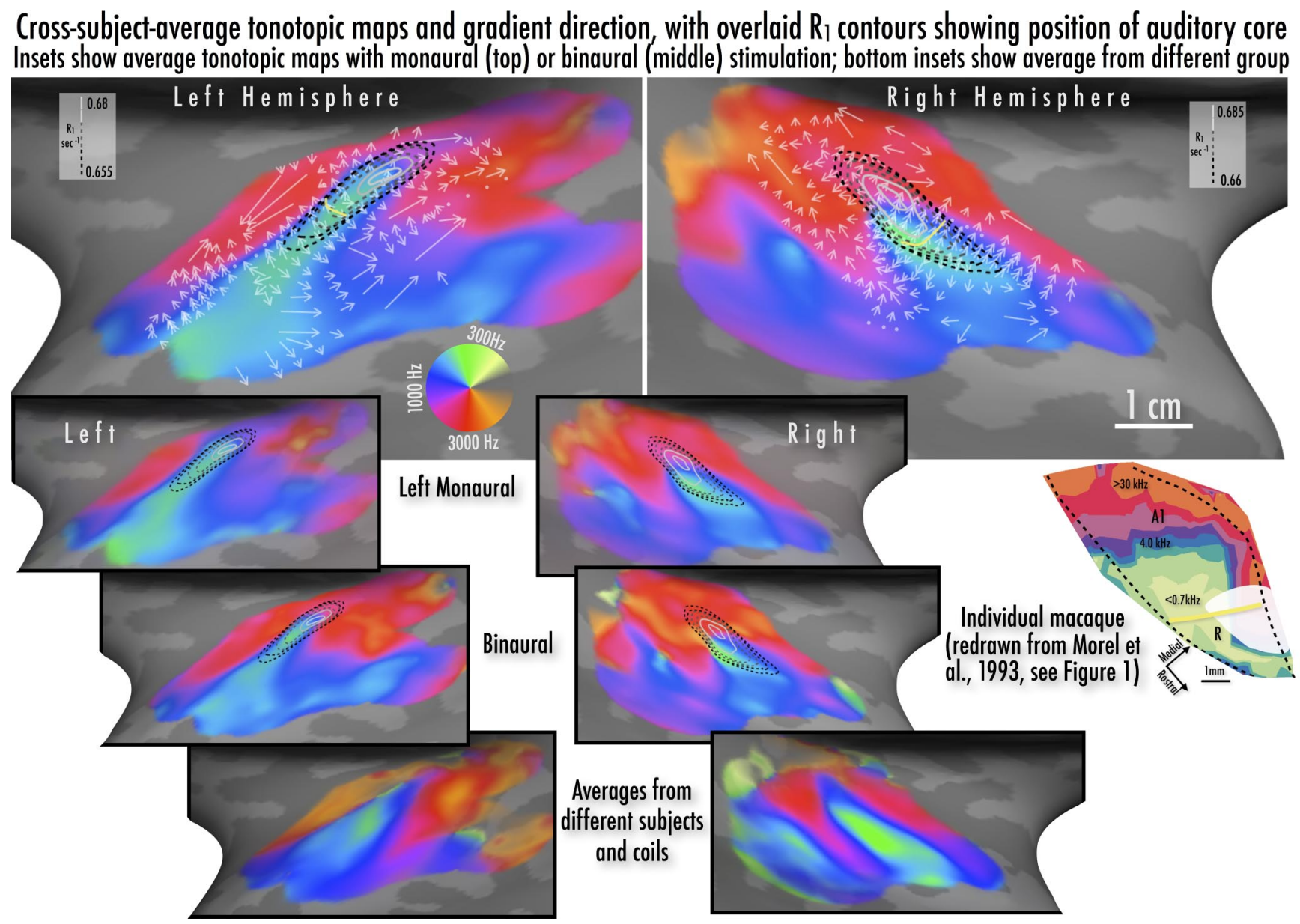

Figure 5. Combined tonotopic maps, gradients, and $\mathrm{R}_{1}$ contours from group averages. Color map shows fMRI results for characteristic frequency with logarithmic scaling in hertz around the color wheel. The angle of the overlaid white arrows shows the tonotopic gradient direction from low-to-high frequency preference at $\sim 1 / 3$ octave steps within tonotopic maps (perpendicular to isofrequency contour), with arrow length reflecting the approximate magnification factor (reciprocal of gradient vector). Dots indicate points where the gradient direction was unclear or interrupted. Dashed lines show $R_{1}$ values in grayscale-coded steps of $0.005 \mathrm{~s}^{-1}$ (same data as Fig. 4); curved yellow line is suggested $A 1 / R$ border based on presence of low-frequency reversal and $R_{1}$ contour. Top and middle inset figures show tonotopic averages with left monaural and binaural stimulation from the same subjects as in the combined average. Bottom inset shows comparison tonotopic averages from different sets of participants and experimental setups; all tonotopic maps were identically masked using the independent auditory localizer (see Materials and Methods).

\section{Individual $R_{1}$ and tonotopic maps}

Since group average tonotopic maps could potentially obscure individual differences, we also assessed individual maps relative to the position of auditory core (Fig. 6) and describe here four qualitative features of the average map (and of macaque monkey maps) that can be found in most individual subjects. A fifth feature involving correlations between tonotopy and $\mathrm{R}_{1}$ is actually more obvious in individuals. For each feature, we list the number of occurrences by hemisphere (N.B.: left hemisphere results are less robust because of fewer runs and less optimal receive coil positioning; also, S4 was excluded on myeloarchitectonic grounds for showing no clear core). The five features are as follows: (1) a corona of higher characteristic frequencies around the medial cap of core $(\operatorname{rh~} N=5 / 6, \operatorname{lh} N=4 / 5)$; (2) an elongated high-frequency patch adjoining the anteromedial edge of the core, transitioning to lower frequencies moving anteriorly (rh $N=6 / 6$, lh $N=5 / 5$ ); (3) a mid-core trough of lowest characteristic frequencies (rh $N=6 / 6$, lh $N=5 / 5$ ); (4) a high-tomedium-to-low progression lateral to the posterior two-thirds of the core (rh $N=6 / 6, \operatorname{lh} N=4 / 5)$; and (5) a transition from $\sim 2 \mathrm{kHz}$ to $1 \mathrm{kHz}$ to $\sim 0.5 \mathrm{kHz}$ characteristic frequency running from the anteromedial edge posterolaterally, with the $\sim 0.5 \mathrm{kHz}$ characteristic frequency region in the highest $\mathrm{R} 1$ region (highest $2-3$ contour lines in each participant) ( $\mathrm{rh} N=5 / 6, \mathrm{lh} N=4 / 5)$.
It is also notable that, unlike the group average tonotopic map, the posteromedial aspect of core reaches into regions with the highest CF in individual maps; in addition, several participants showed a more extensive low to higher frequency progressing anterolaterally to the tip of core (as has been reported for some macaques, e.g., case $91-13$ of Morel et al., 1993). To assess the stability of an individual's tonotopic maps, we compared repeated sessions (four runs each) on one individual (Fig. 7), and found very similar patterns of results for the three sessions using bandpass-swept vocalizations and one session with bandpassswept music; the session using the same experimental setup but with bandpass-swept amplitude-modulated white noise showed similar but much weaker maps. These results were in keeping with the robust maps shown in previous human tonotopy studies (Formisano et al., 2003; Talavage et al., 2004; Woods et al., 2009, 2011; Langers and van Dijk, 2012), in particular the consistency over individual runs and sessions (Humphries et al., 2010).

\section{Summary of results}

Using quantitative anatomical $R_{1}\left(=1 / T_{1}\right)$ maps as myelin markers, we found a small keyhole-shaped area running over the medial two-thirds of Heschl's gyrus that corresponded to nonhuman primate and human postmortem descriptions of 


\section{All individual participants' tonotopic maps (SI-S6) overlaid with 'dethickened' and 'decurved' $R_{1}$ contour}

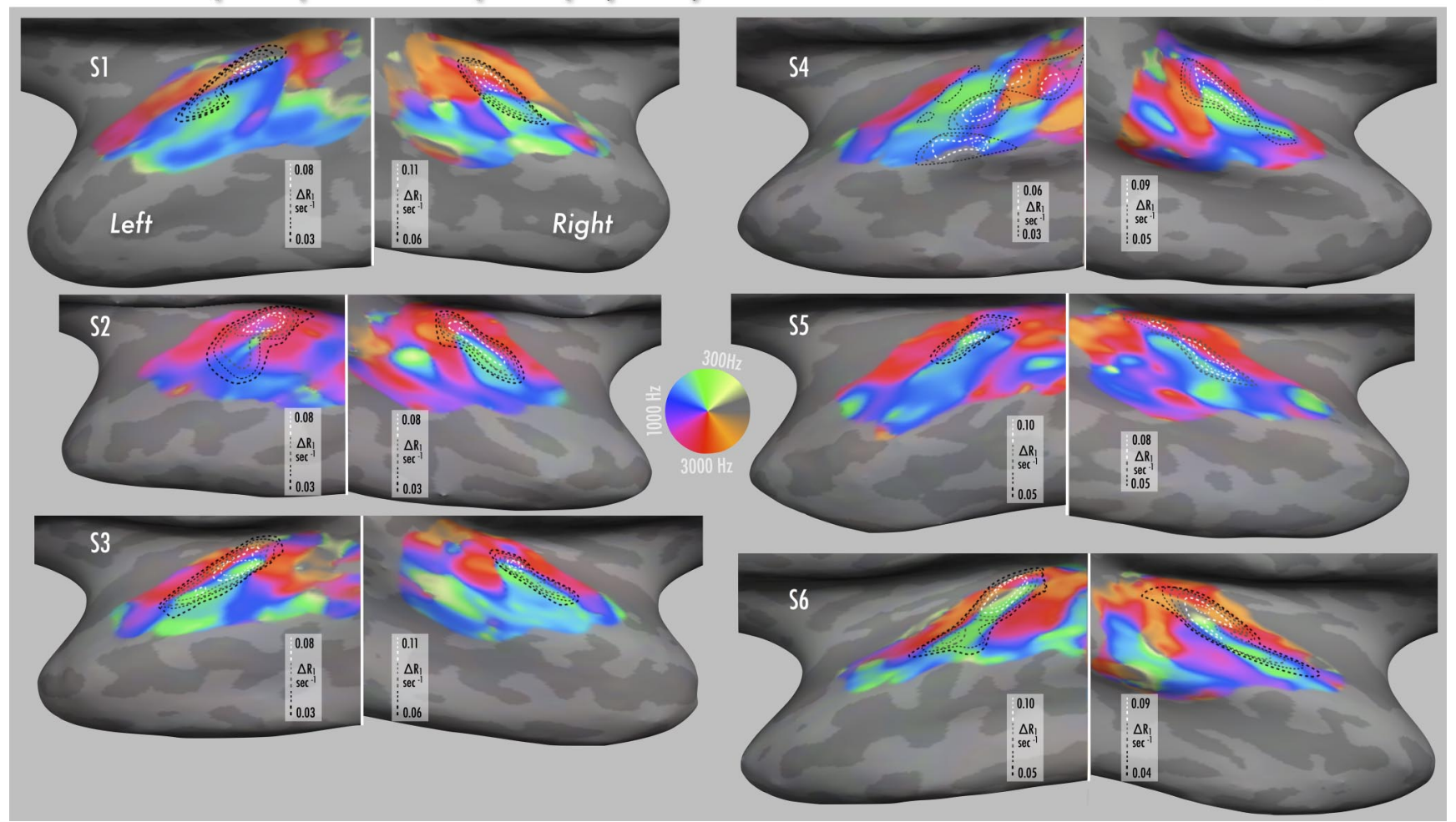

Figure 6. Montage of individual participants' tonotopic maps and $R_{1}$ contours, sampled halfway through cortex and detrended for effects of local curvature and thickness (see Materials and Methods and Fig. 3d). Tonotopic color maps are as in Figure 5. For visual clarity, contours indicate stepwise progression of $R_{1}$ values in probable auditory core only; see heat scale images in Figure 4 for raw $R_{1}$ data for each individual.

Repeated tonotopy sessions of a single participant, monaural presentation to left ear, 32-channel head coil, inflated right hemisphere shown. Top row, filtered vocalization stimulus, each panel from a different session. Bottom row, filtered music (left) and filtered white noise (right)
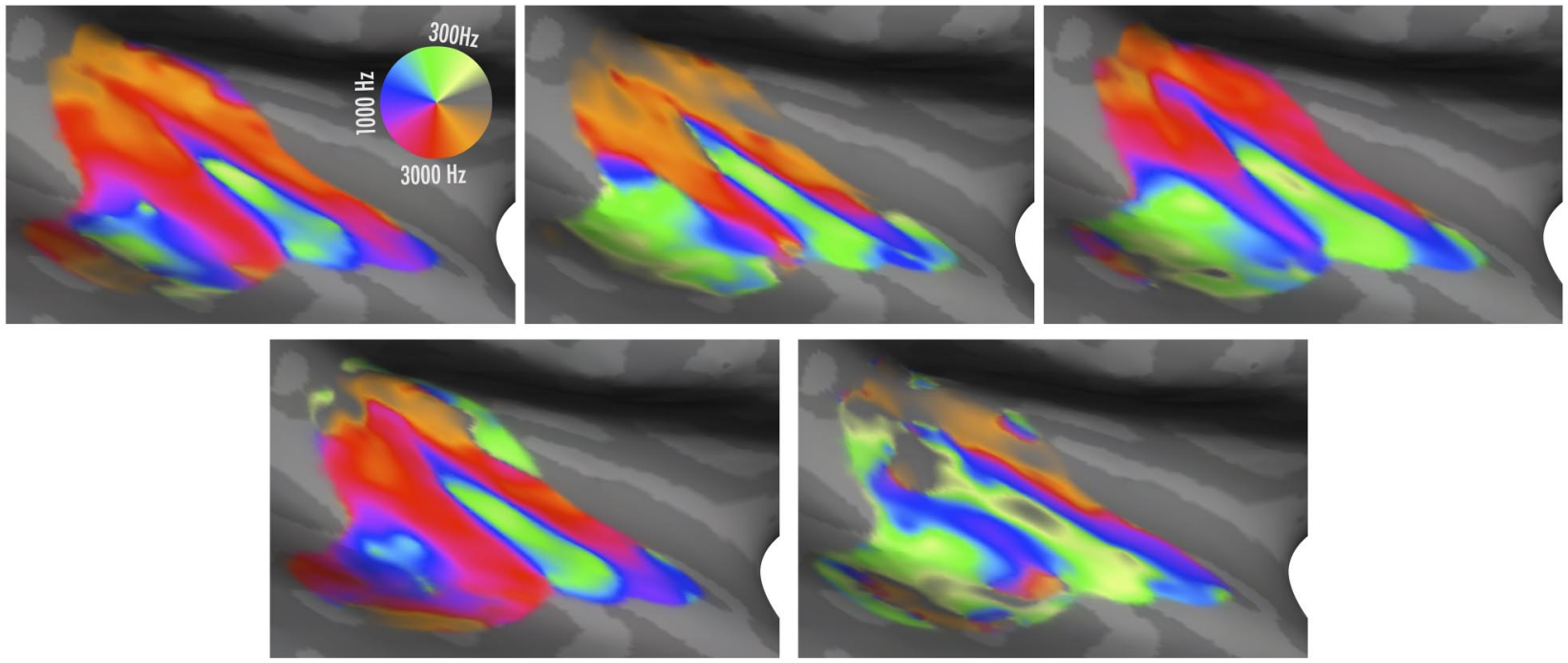

Figure 7. Functional tonotopic maps of an individual subject with focus on the right hemisphere of the inflated superior temporal lobe. Top row shows results from three different sessions of tonotopy using bandpass-swept vocalization stimuli (four runs each); bottom left inset shows one session using bandpass-filtered music (four runs); and bottom right shows one session using bandpass-filtered, amplitude-modulated white noise (four runs).

auditory core (Hopf, 1951; Jones et al., 1995; Hutsler and Gazzaniga, 1996; Kosaki et al., 1997; Hackett et al., 1998, 2001; Morosan et al., 2001; Wallace et al., 2002; Fullerton and Pandya, 2007; Hackett and de la Mothe, 2009) in terms of its laminar profile, orientation, shape, and intensity differences.
This was true both in the surface-based group average as well as in most individual participants. This pattern was consistent across scans and subjects, and was essentially unchanged when the influence of local thickness and curvature on myelination and $\mathrm{R}_{1}$ measures was taken into account (Sereno et al., 2012). 
On both a group and individual level, tonotopic mapping of this core region showed a pattern consistent with previous electrophysiological and architectonic studies in macaques and owl monkeys, with a progression from high-to-low frequency preference moving medially to laterally within the most densely myelinated part of the core. We suggest that this region corresponds to area A1. Within the rostrolateral slightly less densely myelinated finger of the core, there is a low-to-medium frequency progression. Based on similarities with studies in nonhuman primates (Merzenich and Brugge, 1973; Imig et al., 1977; Morel et al., 1993) we suggest this region is the homolog of area R. It is also notable that the gradient field map position associated with A1 and R (Formisano et al., 2003; Fig. 4) appears to be similar to the layout suggested by the $\mathrm{R}_{1}$-defined core and tonotopic maps. The small low-to-medium-to-high frequency gradient within $\mathrm{R}$ is more robust in individual participants than in the group average, probably a result of blurring due to anatomical intersubject variation in this small area. Nonetheless, further high-resolution tonotopic imaging combined with quantitative $\mathrm{R}_{1}$ mapping will be useful in clarifying the group average result, particularly as it is not entirely as expected given some current models of tonotopic mapping in macaques and humans (Baumann et al., 2010; Woods et al., 2009).

\section{Discussion}

These results show that noninvasive in vivo MRI techniques in humans can be used to characterize basic auditory functional regionalization originally revealed in nonhuman primates via a labor-intensive combination of physiological recording followed by postmortem cortical flattening and histology. Since postrecording histology is often partly compromised (Sincich and Horton, 2005), the definition of auditory core boundaries has also relied on other physiological criteria such as tuning bandwidth or firing rate/latency (Recanzone et al., 2000). The current study demonstrates for the first time concordance between tonotopy and myelination on the surface of the human cortex comparable to earlier studies in macaque and owl monkeys (Merzenich and Brugge, 1973; Imig et al., 1977; Pfingst and O'Connor, 1981; Morel and Kaas, 1992; Morel et al., 1993), a result that should help to narrow down the location of the auditory core in humans (Humphries et al., 2010). The current results were consistent across subjects, sessions, auditory stimulus setups, and scanner field strength. Given that the combined functional and structural protocol can be acquired using standard scanner hardware within $\sim 1 \mathrm{~h}$, these techniques should be useful for defining primary auditory fields for further study (for a similar approach, see Talkington et al., 2012) as well as investigations of clinical populations with atypical auditory processes, such as congenital deafness (Karns et al., 2012) or in patients with schizophrenia or tinnitus.

One notable finding is the small size of the core and its constituent areas $\mathrm{A} 1$ and $\mathrm{R}$, compared with that of primary visual and somatosensory cortices (noted also in macaque by Merzenich and Brugge, 1973). Even when borders are liberally defined, the surface area of the core itself rarely exceeds $\sim 2 \mathrm{~cm}^{2}$ across individuals (Fig. 4), with Al occupying somewhat more than half of that area. These dimensions are comparable to those reported for auditory core in human postmortem studies $(\sim 2 \mathrm{~cm} \times \sim 0.9 \mathrm{~cm}$, as in Wallace et al., 2002, their Fig. 6a), after corrections for shrinkage, distortion, and unfolding method.

One open question for identifying auditory core with either $\mathrm{R}_{1}$ maps or postmortem histology is how to set intensity thresholds, particularly at the medial and lateral extents. Borders based on postmortem myelin staining methods tend to be defined by eye, with a sudden change in stain density corresponding to areal edges (after correcting for section-to-section intensity differences), which can then be correlated with borders derived from other measures such as laminar-specific patterns of Nissl concentrations. However, there are large differences in myelination within the core (high medially, decreasing smoothly moving laterally), making a fixed threshold difficult to apply. For instance, as can be seen in Hackett et al., 1998 (their Fig. 2c and d), the grayscale level in the thin, lateral-most part of the core is very similar to that in the belt region surrounding medial core.

In the present MRI-based in vivo study, an analogous case of equivalent $R_{1}$ values at the lateral tip of the core and the region surrounding medial core can be observed in participant S5 (Fig. 4). We have addressed this issue by presenting both the graded and unmasked heat scale data (Figs. $2-4$ ), along with the $\mathrm{R}_{1}$ contour plots (Figs. 5, 6), where low $R_{1}$ values well outside the probable contiguous boundaries of the core are not shown. Gradient-based approaches potentially avoid fixed threshold problems, but the gradient is a local measure that is noisy with unsmoothed data yet highly sensitive to spatial smoothing parameters.

The current findings confirm several results and predictions of the pioneering study of Sigalovsky et al. (2006), who used a multiscan whole-brain FLASH protocol (with different flip angles for each scan) to map $R_{1}$ variation across auditory cortex (averaged over cortical depth), finding consistent patterns of higher $\mathrm{R}_{1}$ values in medial Heschl's gyrus in individual participants, along with hyperintensities along the superior temporal gyrus and planum temporale. As predicted by Sigalovsky et al. (2006), higher resolution $\left[0.512 \mathrm{~mm}^{3}\right.$ voxel volume in the present study vs $1.69 \mathrm{~mm}^{3}$ in Sigalovsky et al. (2006)] and increased SNR (due to 32-channel coil, multi-echo readout, $3 \mathrm{~T}$ field strength, more data points) made it possible to see laminar differences in $R_{1}$ between probabilistically defined core and adjacent regions in the present study (Fig. $2 a$ ). The increased resolution and SNR also enabled us to resample and render $R_{1}$ values at specific cortical depths where core and noncore regions are most different. This sharpened the resulting maps and revealed a correlation between cortical curvature and myelination (Annese et al., 2004) at middle cortical depths (Sereno et al., 2012). Note that the average $R_{1}$ values of $\sim 0.75 \mathrm{~s}^{-1}$ measured by Sigalovsky et al. (2006) at $1.5 \mathrm{~T}$ cannot be directly compared to the average value of $0.69 \mathrm{~s}^{-1}$ within TE1.0 measured in this study at $3 \mathrm{~T}$, since $\mathrm{R}_{1}$ decreases with field strength (Oros-Peusquens et al., 2008). However, the rather small $\mathrm{R}_{1}$ measured at $1.5 \mathrm{~T}$ suggests that it might have been affected by partial volume effects, particularly contamination with CSF, whereas the $\mathrm{R}_{1}$ measured in this study is in line with typical $\mathrm{R}_{1}$ values of gray matter at $3 \mathrm{~T}$ (Oros-Peusquens et al., 2008).

The present study showed that the size and shape of the high- $\mathrm{R}_{1}$ core varies subtly between hemispheres within one individual (Fig. 4, S1 and S6) and more markedly among individuals. In addition, the absolute $\mathrm{R}_{1}$ values in and around the core vary. We think that most of these differences reflect real anatomical intraindividual and interindividual differences. However, some may be due to technical limitations. For example, image artifacts due to head motion most likely caused misestimates of $R_{1}$ in participant S4, obscuring the hyperintensity in the auditory core of the left hemisphere and causing anomalies in the temporal lobe, as well as around highly myelinated areas such as primary motor and somatosensory areas. Quantitative mapping combining data from multiple image acquisitions and with higher than usual resolution requires extra vigilance to reduce head motion 
artifacts. Shortening of the acquisition time would help to reduce motion artifacts within and across the runs, but sufficient SNR must be preserved to preserve $\mathrm{R}_{1}$ map precision.

Inaccuracies in the reconstruction of the white or pial surfaces originating in local misclassification of tissue types (and thus local misestimation of cortical thickness and depth) is another potential source of artifacts. Since myelination (and $R_{1}$ ) vary as much with depth as they do tangentially, this can obscure areal boundaries. Surface imperfections can occur near large pial vessels, where the pial surface closely approaches itself, or where the gray/white matter surface closely approaches itself along thin strands of white matter under small gyri (although it should be noted that these comprise only a tiny fraction of temporal cortex).

Another unexpected finding was that the medial, highfrequency edge of A1 appeared to extend slightly medially $(\sim 3$ $\mathrm{mm}$ ) beyond the boundary of highest $\mathrm{R}_{1}$ values in the group average, and in some individuals. This possible overestimated extent in the group average result may be due in part to blurring over somewhat variable functional anatomy, but may also be due to inherent difficulties in mapping of the BOLD response at the boundary between medial Heschl's gyrus and the deep, narrow circular sulcus. This region is highly vascularized, which may blur the BOLD response where primary cortex adjoins the CM auditory field. This could be resolved by tonotopic mapping at higher field strengths and higher resolution (Formisano et al., 2003; Humphries et al., 2010; Da Costa et al., 2011) or by using acquisition methods thought to suppress contributions from larger vessels (e.g., spin-echo EPI at 7 T; Yacoub et al., 2005). However, this finding may also reflect true physiological and anatomical properties. CM, a very small auditory area that shares the medial high-frequency border with A1, shows much broader frequency tuning, and a more discontinuous tonotopic map (Kajikawa et al., 2005). CM is also highly myelinated, making it difficult to distinguish the A1/CM border based on myeloarchitectonics alone, even in postmortem assays, where changes in cell type are a more unambiguous marker of the A1/CM border.

Our new quantitative, high-resolution, multimodal definition of the auditory core will make it possible to investigate how musical expertise or auditory acuity are related to differences in myelination of A1 and R. (See Duncan and Boynton, 2003, 2007; Schwarzkopf et al., 2011; and Song et al., 2011 for related studies in the visual, auditory, and somatosensory systems.) Our methods can also be extended to nonhuman primates to improve our understanding of homologies as well as potential differences. Studies of auditory and language function in individuals whose core and primary auditory fields are precisely mapped will improve our understanding of the contribution of these early areas to perceptual and cognitive processes. Finally, the sensitive quantitative indices of cortical microstructure developed here may help with early diagnosis of disease and serve as a biomarker to assess the effectiveness of new therapies.

\section{Notes}

A supplemental audio file is available at http://www.bbk.ac.uk/ psychology/videos/FDick/SampleTonotopyStimulus.wav. This includes an example from one channel of a single stimulus sweep (one run would consist of eight such $1 \mathrm{~min} 4 \mathrm{~s}$ sweeps in succession). Note that filtering and perceived loudness are optimized for a particular Sensimetrics earbud within the scanner. This material has not been peer reviewed.

\section{References}

Annese J, Pitiot A, Dinov ID, Toga AW (2004) A myelo-architectonic method for the structural classification of cortical areas. Neuroimage 21: 15-26. CrossRef Medline

Baumann S, Griffiths TD, Rees A, Hunter D, Sun L, Thiele A (2010) Characterisation of the BOLD response time course at different levels of the auditory pathway in non-human primates. Neuroimage 50:1099-1108. CrossRef Medline

Belin P, Fillion-Bilodeau S, Gosselin F (2008) The Montreal Affective Voices: a validated set of nonverbal affect bursts for research on auditory affective processing. Behavior research methods 40:531-539. CrossRef Medline

Bridge H, Clare S (2006) High-resolution MRI: in vivo histology? Philos Trans R Soc Lond B Biol Sci 361:137-146. CrossRef Medline

Cox RW (2012) AFNI: what a long strange trip it's been. Neuroimage. 62: 743-747. CrossRef Medline

Da Costa S, van der Zwaag W, Marques JP, Frackowiak RS, Clarke S, Saenz M (2011) Human primary auditory cortex follows the shape of Heschl's gyrus. J Neurosci 31:14067-14075. CrossRef Medline

Dale AM, Fischl B, Sereno MI (1999) Cortical surface-based analysis. I. Segmentation and surface reconstruction. Neuroimage 9:179-194. CrossRef Medline

Draganski B, Ashburner J, Hutton C, Kherif F, Frackowiak RS, Helms G, Weiskopf N (2011) Regional specificity of MRI contrast parameter changes in normal ageing revealed by voxel-based quantification (VBQ). Neuroimage 55:1423-1434. CrossRef Medline

Duncan RO, Boynton GM (2003) Cortical magnification within human primary visual cortex correlates with acuity thresholds. Neuron 38:659671. CrossRef Medline

Duncan RO, Boynton GM (2007) Tactile hyperacuity thresholds correlate with finger maps in primary somatosensory cortex (S1). Cereb Cortex 17:2878-2891. CrossRef Medline

Engel SA, Rumelhart DE, Wandell BA, Lee AT, Glover GH, Chichilnisky EJ, Shadlen MN (1994) fMRI of human visual cortex. Nature 369:525. CrossRef Medline

Fischl B, Dale AM (2000) Measuring the thickness of the human cerebral cortex from magnetic resonance images. Proc Natl Acad Sci U S A 97: 11050-11055. CrossRef Medline

Fischl B, Sereno MI, Tootell RB, Dale AM (1999) High-resolution intersubject averaging and a coordinate system for the cortical surface. Hum Brain Mapp 8:272-284. CrossRef Medline

Formisano E, Kim DS, Di Salle F, van de Moortele PF, Ugurbil K, Goebel R (2003) Mirror-symmetric tonotopic maps in human primary auditory cortex. Neuron 40:859-869. CrossRef Medline

Fullerton BC, Pandya DN (2007) Architectonic analysis of the auditoryrelated areas of the superior temporal region in human brain. J Comp Neurol 504:470-498. CrossRef Medline

Glasser MF, Van Essen DC (2011) Mapping human cortical areas in vivo based on myelin content as revealed by t1- and t2-weighted MRI. J Neurosci 31:11597-11616. CrossRef Medline

Hackett TA (2007) Organization and correspondence of the auditory cortex of humans and nonhuman primates. In: Evolution of the nervous system (Kaas, JH), pp 109-119. Oxford, UK: Elsevier.

Hackett TA (2011) Information flow in the auditory cortical network. Hear Res 271:133-146. CrossRef Medline

Hackett TA, de la Mothe LA (2009) Regional and laminar distribution of the vesicular glutamate transporter, VGluT2, in the macaque monkey auditory cortex. J Chem Neuroanat 38:106-116. CrossRef Medline

Hackett TA, Stepniewska I, Kaas JH (1998) Subdivisions of auditory cortex and ipsilateral cortical connections of the parabelt auditory cortex in macaque monkeys. J Comp Neurol 394:475-495. CrossRef Medline

Hackett TA, Preuss TM, Kaas JH (2001) Architectonic identification of the core region in auditory cortex of macaques, chimpanzees, and humans. J Comp Neurol 441:197-222. CrossRef Medline

Hagler DJ Jr, Saygin AP, Sereno MI (2006) Smoothing and cluster thresholding for cortical surface-based group analysis of fMRI data. Neuroimage 33:1093-1103. CrossRef Medline

Hagler DJ Jr, Riecke L, Sereno MI (2007) Parietal and superior frontal visuospatial maps activated by pointing and saccades. Neuroimage 35:15621577. CrossRef Medline

Helms G, Dathe H, Dechent P (2008a) Quantitative FLASH MRI at 3T using 
a rational approximation of the Ernst equation. Magn Reson Med 59:667672. CrossRef Medline

Helms G, Finsterbusch J, Weiskopf N, Dechent P (2008b) Rapid radiofrequency field mapping in vivo using single-shot STEAM MRI. Magn Reson Med 60:739-743. CrossRef Medline

Helms G, Draganski B, Frackowiak R, Ashburner J, Weiskopf N (2009) Improved segmentation of deep brain grey matter structures using magnetization transfer (MT) parameter maps. Neuroimage 47:194-198. CrossRef Medline

Hopf A (1951) Die Myeloarchitektonik des Isocortex temporalis beim Menschen. J für Hirnforschung 2:208-279.

Humphries C, Liebenthal E, Binder JR (2010) Tonotopic organization of human auditory cortex. Neuroimage 50:1202-1211. CrossRef Medline

Hutsler JJ, Gazzaniga MS (1996) Acetylcholinesterase staining in human auditory and language cortices: regional variation of structural features. Cereb Cortex 6:260-270. CrossRef Medline

Imig TJ, Ruggero MA, Kitzes LM, Javel E, Brugge JF (1977) Organization of auditory cortex in the owl monkey (Aotus trivirgatus). J Comp Neurol 171:111-128. CrossRef Medline

Jones EG, Dell'Anna ME, Molinari M, Rausell E, Hashikawa T (1995) Subdivisions of macaque monkey auditory cortex revealed by calciumbinding protein immunoreactivity. J Comp Neurol 362:153-170. CrossRef Medline

Josephs O, Dick F, Sereno M, Weiskopf N (2009) An isodynamic high fidelity MRI compatible headphone. Neuroimage 47:S186. CrossRef

Kajikawa Y, de La Mothe L, Blumell S, Hackett TA (2005) A comparison of neuron response properties in areas $\mathrm{A} 1$ and $\mathrm{CM}$ of the marmoset monkey auditory cortex: tones and broadband noise. J Neurophysiol 93:22-34. Medline

Karns CM, Dow MW, Neville HJ (2012) Altered cross-modal processing in the primary auditory cortex of congenitally deaf adults: a visualsomatosensory FMRI study with a double-flash illusion. J Neurosci 32: 9626-9638. CrossRef Medline

Konen CS, Behrmann M, Nishimura M, Kastner S (2011) The functional neuroanatomy of object agnosia: a case study. Neuron 71:49-60. CrossRef Medline

Kosaki H, Hashikawa T, He J, Jones EG (1997) Tonotopic organization of auditory cortical fields delineated by parvalbumin immunoreactivity in macaque monkeys. J Comp Neurol 386:304-316. CrossRef Medline

Langers DR, van Dijk P (2012) Mapping the tonotopic organization in human auditory cortex with minimally salient acoustic stimulation. Cereb Cortex 22:2024-2038. CrossRef Medline

Larsson J, Heeger DJ (2006) Two retinotopic visual areas in human lateral occipital cortex. J Neurosci 26:13128-13142. CrossRef Medline

Lutti A, Hutton C, Finsterbusch J, Helms G, Weiskopf N (2010) Optimization and validation of methods for mapping of the radiofrequency transmit field at 3T. Magn Reson Med 64:229-238. CrossRef Medline

Lutti A, Stadler J, Josephs O, Windischberger C, Speck O, Bernarding J, Hutton C, Weiskopf N (2012) Robust and fast whole brain mapping of the RF transmit field B1 at 7T. PLoS One 7:e32379. CrossRef Medline

Merzenich MM, Brugge JF (1973) Representation of the cochlear partition of the superior temporal plane of the macaque monkey. Brain Res 50:275296. CrossRef Medline

Morel A, Kaas JH (1992) Subdivisions and connections of auditory cortex in owl monkeys. J Comp Neurol 318:27-63. CrossRef Medline

Morel A, Garraghty PE, Kaas JH (1993) Tonotopic organization, architectonic fields, and connections of auditory cortex in macaque monkeys. J Comp Neurol 335:437-459. CrossRef Medline

Morosan P, Rademacher J, Schleicher A, Amunts K, Schormann T, Zilles K (2001) Human primary auditory cortex: cytoarchitectonic subdivisions and mapping into a spatial reference system. Neuroimage 13:684-701. CrossRef Medline

Oros-Peusquens AM, Laurila M, Shah NJ (2008) Magnetic field dependence of the distribution of NMR relaxation times in the living human brain. MAGMA 21:131-147. CrossRef

Petkov CI, Kayser C, Augath M, Logothetis NK (2006) Functional imaging reveals numerous fields in the monkey auditory cortex. PLoS Biol 4:e215. CrossRef Medline

Pfingst BE, O'Connor TA (1981) Characteristics of neurons in auditory cortex of monkeys performing a simple auditory task. J Neurophysiol 45:1634. Medline

Preibisch C, Deichmann R (2009) Influence of RF spoiling on the stability and accuracy of T1 mapping based on spoiled FLASH with varying flip angles. Magn Reson Med 61:125-135. CrossRef Medline

Rajimehr R, Tootell RB (2009) Does retinotopy influence cortical folding in primate visual cortex? J Neurosci 29:11149-11152. CrossRef Medline

Recanzone GH, Schreiner CE, Sutter ML, Beitel RE, Merzenich MM (1999) Functional organization of spectral receptive fields in the primary auditory cortex of the owl monkey. J Comp Neurol 415:460-481. CrossRef Medline

Recanzone GH, Guard DC, Phan ML (2000) Frequency and intensity response properties of single neurons in the auditory cortex of the behaving macaque monkey. J Neurophysiol 83:2315-2331. Medline

Saygin AP, Sereno MI (2008) Retinotopy and attention in human occipital, temporal, parietal, and frontal cortex. Cereb Cortex 18:2158-2168. Medline

Schwarzkopf DS, Song C, Rees G (2011) The surface area of human V1 predicts the subjective experience of object size. Nat Neurosci 14:28-30. CrossRef Medline

Sereno MI, Huang RS (2006) A human parietal face area contains aligned head-centered visual and tactile maps. Nat Neurosci 9:1337-1343. CrossRef Medline

Sereno MI, Dale AM, Reppas JB, Kwong KK, Belliveau JW, Brady TJ, Rosen BR, Tootell RB (1995) Borders of multiple visual areas in humans revealed by functional magnetic resonance imaging. Science 268:889-893. CrossRef Medline

Sereno MI, Lutti A, Weiskopf N, Dick F (2012) Mapping the human cortical surface by combining quantitative T1 with retinotopy. Cereb Cortex. Advance online publication. Retrieved October 24, 2012. doi: 10.1093/cercor/bhs213. CrossRef

Sigalovsky IS, Fischl B, Melcher JR (2006) Mapping an intrinsic MR property of gray matter in auditory cortex of living humans: a possible marker for primary cortex and hemispheric differences. Neuroimage 32:1524-1537. CrossRef Medline

Sincich LC, Horton JC (2005) The circuitry of V1 and V2: integration of color, form, and motion. Annu Rev Neurosci 28:303-326. CrossRef Medline

Song C, Schwarzkopf DS, Kanai R, Rees G (2011) Reciprocal anatomical relationship between primary sensory and prefrontal cortices in the human brain. J Neurosci 31:9472-9480. CrossRef Medline

Talavage TM, Sereno MI, Melcher JR, Ledden PJ, Rosen BR, Dale AM (2004) Tonotopic organization in human auditory cortex revealed by progressions of frequency sensitivity. J Neurophysiol 91:1282-1296. Medline

Talkington WJ, Rapuano KM, Hitt LA, Frum CA, Lewis JW (2012) Humans mimicking animals: a cortical hierarchy for human vocal communication sounds. J Neurosci 32:8084-8093. CrossRef Medline

Thesen S, Heid O, Mueller E, Schad LR (2000) Prospective acquisition correction for head motion with image-based tracking for real-time fMRI. Magn Reson Med 44:457-465. CrossRef Medline

Trampel R, Ott DV, Turner R (2011) Do the congenitally blind have a stria of Gennari? First intracortical insights in vivo. Cereb Cortex 21:2075-2081. CrossRef Medline

Wallace MN, Johnston PW, Palmer AR (2002) Histochemical identification of cortical areas in the auditory region of the human brain. Exp Brain Res 143:499-508. CrossRef Medline

Walters NB, Egan GF, Kril JJ, Kean M, Waley P, Jenkinson M, Watson JD (2003) In vivo identification of human cortical areas using highresolution MRI: an approach to cerebral structure-function correlation. Proc Natl Acad Sci U S A 100:2981-2986. CrossRef Medline

Weiskopf N, Lutti A, Helms G, Novak M, Ashburner J, Hutton C (2011) Unified segmentation based correction of R1 brain maps for RF transmit field inhomogeneities (UNICORT). Neuroimage 54:2116-2124. CrossRef Medline

Wiggins GC, Triantafyllou C, Potthast A, Reykowski A, Nittka M, Wald LL (2006) 32-channel 3 Tesla receive-only phased-array head coil with soccer-ball element geometry. Magn Reson Med 56:216-223. CrossRef Medline

Woods DL, Stecker GC, Rinne T, Herron TJ, Cate AD, Yund EW, Liao I, Kang X (2009) Functional maps of human auditory cortex: effects of acoustic features and attention. PLoS One 4:e5183. CrossRef Medline

Woods DL, Herron TJ, Cate AD, Kang X, Yund EW (2011) Phonological processing in human auditory cortical fields. Front Hum Neurosci 5:42. Medline

Yacoub E, Van De Moortele PF, Shmuel A, Uğurbil K (2005) Signal and 
noise characteristics of Hahn SE and GE BOLD fMRI at $7 \mathrm{~T}$ in humans. Neuroimage 24:738-750. CrossRef Medline
Zilles K, Amunts K (2010) Centenary of Brodmann's map-conception and fate. Nat Rev Neurosci 11:139-145. CrossRef Medline 